\title{
Does selegiline delay progression of Parkinson's disease? A critical re-evaluation of the DATATOP study
}

\author{
Christopher D Ward
}

\begin{abstract}
The deprenyl and tocopherol antioxidative treatment (DATATOP) study has shown that selegiline (deprenyl), with or without tocopherol, reduces physical and psychological deficits in patients with Parkinson's disease within one month of treatment and reduces the probability of reaching a primary endpoint, the decision to treat with levodopa. This paper critically re-evaluates the inference that selegiline has a neuroprotective effect and thus delays progression of Parkinson's disease. Analysis is based on a simple model which assumes that clinically measured impairment is proportional to degree of cellular dysfunction in Parkinson's disease. The analysis suggests that the reduced probability of reaching an endpoint was due to a direct treatment effect rather than to neuroprotection.
\end{abstract}

(F Neurol Neurosurg Psychiatry 1994;57:217-220)

Selegiline ((-)deprenyl) is a monoamine oxidase-B (MAO-B) inhibitor ${ }^{1}$ that might theoretically inhibit the action of a putative toxin or reduce "oxidative stress" on neurons. ${ }^{2-4}$ The sole purpose of this paper is to examine evidence from the deprenyl and tocopherol antioxidative treatment (DATATOP) study ${ }^{5-7}$ that selegiline is "neuroprotective"-that is, it reduces the rate of progression of Parkinson's disease. Despite some controversy ${ }^{8}$ the neuroprotective hypothesis has been widely accepted. ${ }^{9}$

Eight hundred previously untreated patients were randomised to receive either selegiline, tocopherol, tocopherol and selegiline, or placebo. No levodopa or other treatment was given. Treatment effects were found for selegiline but not for tocopherol. The primary endpoint was the decision to initiate levodopa treatment. Other indices of treatment effects were termed secondary response variables and included measures of motor impairment, physical disability, and mental state. Analysis showed that selegiline treatment delayed the primary endpoint by about nine months. Secondary response variables showed improvement at one and three months of treatment ("wash-in" effect), and some deterioration was seen two months after stopping selegiline ("wash-out"). Mean rate of change of these variables was significantly lower in the treatment group.

The study was based on the assumption that when disease progression reaches a threshold, a measurable clinical endpoint occurs as shown schematically in the figure (A). Individual levels of impairment (for example, degree of motor slowing) are distributed about the mean and increase with time. The figure (B) shows the effect of a neuroprotective agent: the rate of change of impairment is reduced. The figure (C) shows a direct treatment effect (such as that of levodopa). The rate of disease progression is unchanged but mean time to endpoint is delayed as a result of a quantal reduction in initial impairment. The model assumes that individual progression rates are roughly parallel. In keeping with this assumption, patients in the DATATOP study who reached the endpoint had higher average levels of baseline impairment and disability than those who did not. Rate of change of secondary response variables was in fact non-linear rather than as shown in the figure, ${ }^{5}$ but a non-linear model would have similar properties.

\section{Influence of study design on interpretation}

In the figure the probability of reaching endpoint is represented by the proportion of subjects above threshold: it is 0.5 at the point where the line representing mean level of impairment crosses the threshold level. In the DATATOP study Kaplan Meier curves were used to plot the probability of reaching the endpoint at intervals after randomisation. The rate at which patients reach the endpoint, and hence the slope of the Kaplan Meier curve, will depend on the speed of progression. The neuroprotective hypothesis predicts that the rate at which subjects reach the endpoint will be reduced, so that the Kaplan Meier curve will have a lower slope in the treated than in the untreated group. The figure (C) shows that a quantal reduction in initial impairment also causes a delay in reaching threshold so that the probability of reaching the endpoint is initially reduced. The underlying speed of progression is unchanged, however. The extent to which the DATATOP study distinguishes neuroprotec- 
Figure Schematic representation of progression of motor impairment in a cohort of untreated patients with Parkinson's disease. Each line represents a single patient. Clinical endpoint occurs at threshold level. (A) Untreated cohort. (B) Effect of neuroprotective treatment. The rate of progression of impairment is reduced and the mean time at which threshold is reached is delayed compared with the untreated group.

(C) Effect of direct ("symptomatic") treatment (for example, with levodopa). A quanta reduction in impairment does not affect rate of progression thereafter but does delay the time at which threshold is reached.
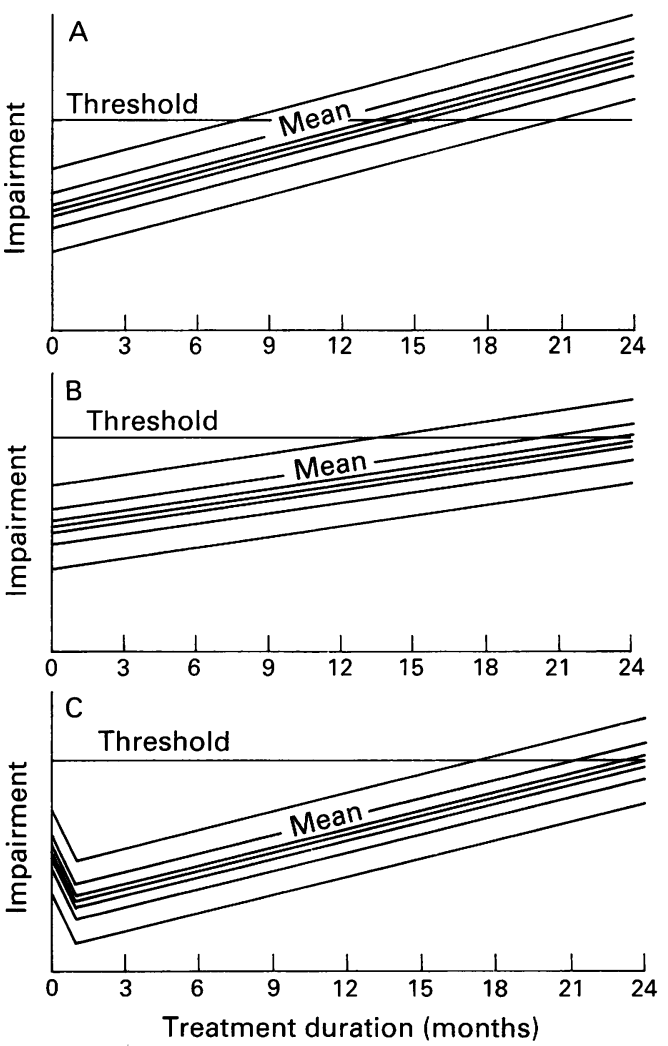

tion from a direct treatment effect depends on two issues related to trial design. Firstly, was disease progression validly measured? Secondly, was duration of follow up adequate?

\section{Was disease progression validly measured?}

The primary endpoint, the need for levodopa treatment, was based on subjectively perceived needs of the patient rather than on objective measurements such as motor speed. The subjective impact of disease is a poor predictor of severity of impairment in neurological disorders ${ }^{10-12}$ and therefore has an uncertain relation with the underlying pathological process. The question under discussion here is the biological efficacy of selegiline as a neuroprotective agent; in this respect DATATOP was an "efficacy" rather than a "pragmatic" trial. ${ }^{13}$ The primary endpoint provided only a weak indication of either the severity or the rate of progression of neuronal dysfunction. To demonstrate the effect shown in the figure (B) it would have been preferable to use reliable, valid, serial measures of biologically based impairments.

Selegiline significantly improved the UPDRS mental subscore at one and three months, and the Hamilton Rating Scale at three months of treatment. ${ }^{57}$ Although in small trials selegiline has not been found to be effective treatment for depression in Parkinson's disease $\mathrm{e}^{1415}$ it does increase feelings of vitality. ${ }^{16}$ It is possible, therefore, that mood elevation could have influenced timing of endpoint without disease progression being affected.
In conclusion, the primary endpoint was subject to several influences and provided only an indirect index of disease progression.

Was duration of follow up adequate?

The model shown in the figure indicates two reasons why an inadequate period of follow up will underestimate the rate of reaching endpoint and therefore overestimate any therapeutic effect. The first consideration is the shape of the probability distribution of impairment: the most severe cases are relatively rare, as shown schematically in the figure. Secondly, as there is a lag period between the time when the first untreated patients pass the endpoint and the time when treated patients begin to do so, the ratio of treated to untreated patients achieving endpoint is small initially. Kaplan Meier curves derived from an untreated cohort as in the figure (A) can only be validly compared with a treated group after a lag period corresponding to the mean delay in reaching the endpoint. This delay was nine months in the DATATOP study. ${ }^{7}$ Analysis performed shortly after this period will be hazardous because of non-linearity and it is therefore desirable to analyse results when follow up has been continued for a period not far short of the projected mean time for treated patients to reach the endpoint; this was 24 months. ${ }^{7}$

As well as overestimating any treatment effect, premature analysis will hinder interpretation of the effect's mechanism. Because an initially low rate of reaching the endpoint in the treatment group may be artefactual, it does not provide support for neuroprotection. Definitive interpretation would require further follow up to ensure that the rate remained lower and that hazard ratios remained stable well beyond the lag period. With the direct treatment effect shown in the figure (C) Kaplan Meier curves would diverge initially but would eventually become parallel.

The first published results of the study were based on an analysis performed when the mean period of follow up was 12 months-that is, only three months longer than the lag period. ${ }^{5}$ Results suggested divergence of Kaplan Meier curves, supporting neuroprotection. In keeping with the predictions outlined, however, subsequent analysis ${ }^{7}$ showed a decline in the estimate of the size of treatment effect. More importantly, Kaplan Meier curves showed divergence only for 18 months of follow up; they were subsequently parallel. This finding could either be due to lack of long term patients or to a direct treatment effect as argued earlier. The more recent analysis does not support neuroprotection.

\section{Evidence for neuroprotection}

In terms of the model shown in the figure, the most important evidence for neuroprotection 
comes from studying the effect of treatment on the rate at which impairment increases over time. As the figure (B) predicts, selegiline treatment reduced the mean rate of change of secondary response variables. The published reports ${ }^{57}$ do not state how this rate was calculated and the data are not fully presented. Interpretation is complicated by the fact that rate of change of variables was nonlinear. This is apparent from the size of the short term wash-in effect and from the statement that most of the deterioration occurred in the period immediately before reaching the endpoint ${ }^{5}$; comparisons of mean rates would not seem to be the ideal form of analysis for non-linear data. There remains one finding ${ }^{7}$ that is supportive of neuroprotection, however, and that is difficult to explain on the basis of a direct treatment effect. Patients who had not reached the endpoint were studied after the two month wash-out period and those treated with selegiline had significantly lower mean rates of decline.

\section{Evidence for a direct treatment effect}

A direct treatment effect as in the figure (C) would predict an early improvement in impairment and this was found: all variables that improved did so significantly within three months of treatment, and most within one month. A further prediction from a direct effect would be that withdrawal of treatment would cause a short term increase in impairment; withdrawal of a neuroprotective agent would have no short term effects. In the earlier publication ${ }^{5}$ patients reaching the endpoint were studied one month after stopping selegiline and wash-out effects were not seen. In the second publication ${ }^{7}$ patients who had not reached endpoint were also studied up to two months after treatment. At two months there was a significant decline in total impairment score and in the motor component. Both wash-in and wash-out studies thus supported a direct treatment effect.

According to the model in the figure (C) a larger direct treatment effect might be associated with a longer period before the endpoint is reached. No such relation was shown. ${ }^{7}$ There was a time lapse of several months, however, between the occurrence of early treatment effects and the primary endpoint and a direct treatment effect might have been less pronounced with time. Interpretation is further complicated by a possibility not illustrated in the figure (C): a larger initial treatment effect could have been correlated with a higher level of impairment and hence with a greater prior probability of reaching endpoint early. Another consideration is that in this large scale clinical study of Parkinson's disease diagnostic error was probably $20 \%$ to $30 \%{ }^{17}$; the error rate is around $24 \%$ when an early, untreated cohort is selected. ${ }^{18}$ Misdiagnosed patients are usually resistant to treatment and their inclusion in group analyses complicates the relation between initial treatment effects and outcome.

\section{Can a small direct treatment affect endpoint?}

Within three months of treatment the treated group had better scores than the untreated group on most scales measuring aspects of disability and motor impairment; mean improvements were small (between $13 \%$ and $23 \%$ of standard deviation) but highly significant. The authors described the direct treatment effect as "slight and not likely to be of clinical consequence". The reduced numbers reaching the endpoint illustrated in the figure (C), however, would be produced by reduction in impairment of less than half a standard deviation if we assume a normal distribution. ${ }^{19}$

Furthermore, the direct treatment effect in individual patients may have been larger than the sample mean values would suggest, having been diluted by either of two factors. Firstly, if improvement were confined to patients with above average levels of baseline impairment, the result shown in the figure (C) could be associated with a mean reduction of less than a quarter of a standard deviation. Secondly, diagnostic error in the sample dilutes observed mean direct treatment effects as misdiagnoses respond poorly to drug treatment (see earlier).

\section{Health economic implications}

A major economic benefit of a neuroprotective agent would be a reduction in severe morbidity at later stages of the disease. ${ }^{2021}$ The DATATOP study provides no indication that reductions in morbidity would be sustained during chronic treatment, however; the lack of washout effect argues against a sustained effect. Although it is suggested that a $10 \%$ reduction in morbidity would produce annual savings in the United States of $\$ 327$ million ( $\$ 1.3$ per head) $)^{22}$ this is only a little more than the current cost of treating all patients with Parkinson's disease with $10 \mathrm{mg}$ selegiline daily, assuming a prevalence of 1.6 per $1000 . .^{23}$ In routine use, misdiagnoses would add substantially to this cost. Conventional therapeutic agents can be used selectively in individual patients and are less expensive than a drug used indiscriminately for all patients carrying the diagnosis of Parkinson's disease.

\section{Conclusion}

The DATATOP study is the largest therapeutic trial ever undertaken in Parkinson's disease and its authors have produced valuable data to suggest that selegiline has direct therapeutic effects in Parkinson's disease, although clinical indications require further study. The size of the direct treatment effect was sufficient to explain the observed reduction in probability of reaching endpoint. There is little evidence that selegiline reduces the rate of progression of Parkinson's disease. A further trial is ethically justified and should be based on measures of underlying disease 
progression with rate of change of impairment as the main treatment criterion. Analysis of such a trial must take account of theoretical considerations that indicate the minimum period of informative follow up.

I am grateful for comments made on an earlier draft by $\mathrm{Dr} M$ Campbell and Professor C George.

1 Johnson S, Sandler M. The pharmacology of selegiline. Rev Contemp Pharmacol 1992;9:51-65.

2 Langston JW. Selegiline as a neuroprotective therapy in Parkinson's disease: Concepts and controversies. Neurology 1990;40:(suppl 3):61-6.

3 Olanow CW. Oxidation reactions in Parkinson's disease. Neurology 1990;40(suppl 3):32-7.

4 Langston JW, Ballard P. Parkinsonism induced by 1-methyl-4-phenyl-1,2,3,6-tetrahydropyridine (MPTP): Implications for treatment and the pathogenesis of Parkinson's disease. Can f Neurol Sci 1984;11(suppl 1): Parkinson.

5 Parkinson Study Group. The effect of selegiline on the progression of disability in early Parkinson's disease. $N$ Engl fMed 1989;321:1364-71.

6 Parkinson Study Group. DATATOP: a multi-center controlled clinical trial in early Parkinson's disease. Arch Neurol 1989;46:1052-60.

7 Parkinson Study Group. Effects of tocopherol and deprenyl on the progression of disability in Parkinson's disease. N Engl F Med 1993;328:176-83.

8 Landau WM. Clinical neuromythology IX. Pyramid sale in the bucket shop: DATATOP bottoms out. Neurology 1990;40:1337-9.

9 Stewart DA, Macphee GJA. New drugs in Parkinson's disease. Hosp Update 1991;17:900-11.
10 World Health Organisation. The international classification of impairments, disabilities, and handicaps-a manual of classification relating to the consequences of disease. Geneva: WHO, 1980.

11 Granger CV. Outcome of comprehensive rehabilitation: an analysis based on the impairment, disability and an analysis based on the impairment, disability and 12 Ward CD Rehabilitation in Parkinson's disease. Rev Clin Gerontol 1992;2:254-68.

13 Schwartz D, Flamant R, Lellouch J. Clinical trials. New York: Academic Press, 1981

14 Shaw JE, Pearce JMS. Selegiline in the management of Parkinson's disease. Rev Contemp Pharmacol 1992;3: 67-76.

15 Lees AJ, Shaw KM, Kohout LJ, et al. Deprenyl in Parkinson's disease. Lancet 1977;2:791-5.

16 Lees AJ. Selegiline hydrochloride and depression. Acta Neurol Scand 1991;136:91-4.

17 Ward CD, Gibb WR. Research diagnostic criteria for Parkinson's disease. Adv Neurol 1990;53:245-9.

18 Hughes AJ, Daniel SE, Kilford L, Lees AJ. Accuracy of clinical diagnosis of Parkinson's disease: a clinicopathological study of 100 cases. F Neurol Neurosurg Psychiatry logical study of

19 Lindley DV, Miller JCP. Table of standardized deviates. Cambridge elementary statistical tables. Cambridge: Cambridge elementary statistical

20 Chrisp P, Mammen GJ, Sorkin EM. Selegiline. A review of its pharmacology, symptomatic benefits and protective potential in Parkinson's disease. Drugs and aging 1991;1:228-48.

21 West R. Parkinson's disease London: Office of Health Economics, 1991;29.

22 Kurlan R, Clark S, Shoulson I, Penney J (The Parkinson Study Group). Economic impact of protective therapy for early Parkinson's disease. Ann Neurol 1988;24:153.

23 Mutch WJ, Digwall-Fordyce I, Downie AW, Paterson JG, Roy SK. Parkinson's disease in a Scottish city. $B M^{7}$ 1986;292:534-6. 